\title{
LC/MS-Based Polar Metabolite Profiling Identified Unique Biomarker Signatures for Cervical Cancer and Cervical Intraepithelial Neoplasia Using Global and Targeted Metabolomics
}

\author{
Imran Khan ${ }^{1,+}{ }^{\dagger}$ Miso Nam ${ }^{2,+}$, Minji Kwon ${ }^{1}$, Sang-soo Seo ${ }^{3}$, Sunhee Jung ${ }^{2}$, Ji Soo Han ${ }^{2}$, \\ Geum-Sook Hwang ${ }^{2, *}$ and Mi Kyung Kim ${ }^{1, *}$ \\ 1 Division of Cancer Epidemiology and Prevention, National Cancer Center, Madu-dong, Ilsandong-gu, \\ Goyang-si, Gyeonggi-do 10408, Korea; imranbbt@ncc.re.kr (I.K.); 74433@ncc.re.kr (M.K.) \\ 2 Integrated Metabolomics Research Group, Western Seoul Center, Korea Basic Science Institute, \\ Seoul 03759, Korea; nms65@kbsi.re.kr (M.N.); jsh204@kbsi.re.kr (S.J.); hjs0503@kbsi.re.kr (J.S.H.) \\ 3 Center for Uterine Cancer, National Cancer Center, Madu-dong, Ilsandong-gu, Goyang-si, \\ Gyeonggi-do 10408, Korea; ssseomd@ncc.re.kr \\ * Correspondence: gshwang@kbsi.re.kr (G.-S.H.); alrud@ncc.re.kr (M.K.K.); Tel.: +82-2-6908-6200 (G.-S.H.); \\ +82-31-920-2202 (M.K.K.); Fax: +82-2-6908-6239 (G.-S.H.); +82-31-920-2006 (M.K.K.) \\ + These authors contributed equally to this work.
}

Received: 14 March 2019; Accepted: 8 April 2019; Published: 10 April 2019

\begin{abstract}
Cervical cancer remains one of the most prevalent cancers among females worldwide. Therefore, it is important to discover new biomarkers for early diagnosis of cervical intraepithelial neoplasia (CIN) and cervical cancer, preferably non-invasive ones. In the present study, we aimed to identify unique metabolic signatures for CINs and cervical cancers using global and targeted metabolomic profiling. Plasma samples (69 normal, 55 CIN1, 42 CIN2/3, and 60 cervical cancer) were examined by ultra-performance liquid chromatography-quadrupole-time-of-flight mass spectrometry (UPLC-QTOF-MS) coupled with multivariate statistical analysis. Metabolic pathways were analyzed using the integrated web-based tool MetaboAnalyst. A multivariate logistic regression analysis was conducted to evaluate the combined association of metabolites and human papillomavirus (HPV) status with the risk of cervical carcinogenesis. A total of 28 metabolites exhibiting discriminating levels among normal, CIN, and cervical cancer patients (Kruskal-Wallis test $p<0.05$ ) were identified in the global profiling analysis. The pathway analysis showed significantly altered alanine, aspartate, and glutamate metabolic pathways (FDR $p$-value $<0.05$ ) in both the discovery and validation phases. Seven metabolites (AMP, aspartate, glutamate, hypoxanthine, lactate, proline, and pyroglutamate) were discriminated between CINs and cervical cancer versus normal (area under the curve (AUC) value $>0.8)$. The levels of these metabolites were significantly high in patients versus normal $(p<0.0001)$ and were associated with increased risk of developing CIN2/3 and cervical cancer. Additionally, elevated levels of the seven metabolites combined with positive HPV status were correlated with substantial risk of cancer progression. These results demonstrated that metabolomics profiling is capable of distinguishing CINs and cervical cancers from normal and highlighted potential biomarkers for the early detection of cervical carcinogenesis.
\end{abstract}

Keywords: biomarker; cervical cancer; human papillomavirus; metabolomic analysis; metabolic pathways; plasma profiling 


\section{Introduction}

Cervical cancer is a common malignant disease in women around the world. It is the third most commonly diagnosed cancer ( 485,000 cases) and the fourth leading cause of cancer-related deaths $(236,000)$ per year worldwide [1,2]. The main cause of cervical cancer is persistent oncogenic human papillomavirus (HPV) infection. Cervical intraepithelial neoplasia (CIN) 1-3 and carcinoma in situ are the precursor lesions of cervical cancer [3]. Other factors such as sexually transmitted infections, oral contraceptive use, smoking status, parity, and diet also contribute to the development of cervical cancer [4]. Patients diagnosed with cervical cancer often show no obvious signs or symptoms at early stages, which fact can lead to misdiagnosis. The incidence of cervical cancer worldwide varies significantly due to advancements in research and precautionary measures taken by developed countries [5]. Incidence decline can be attributed to regular application of Pap tests, which can reveal CINs and carcinoma in situ before they develop into cervical cancer, and vaccination [6]. However, current diagnostics for cervical cancer are based on histological and morphological examinations, which lack sensitivity and specificity [7].

Recent developments in molecular biology, proteomics, and metabolomics have inaugurated a new era marked by the discovery of biomarkers for cervical cancer [7,8]. Metabolomic research for cervical cancer treatment applications, in particular, has attracted immense interest globally. Metabolites, the end products of several biological processes, show potential as accurate biomarkers identifying upstream biological processes such as genetic mutations and environmental changes $[9,10]$. Metabolomics based on platforms such as nuclear magnetic resonance (NMR) spectroscopy or mass spectrometry (MS) combined with chromatography has been utilized for comprehensive analysis and quantification of metabolites in biological systems [11-13]. Metabolomics as applied to cancer research has been a powerful tool for identification of metabolic changes in cancer development and progression and discovery of non-invasive biomarkers for cancer prediction, monitoring, and diagnosis [14-16]. Changes in metabolic profiles and pathways can provide a better understanding of dysregulated metabolism in tumor initiation and progression compared with normal (healthy) controls [17]. A well-known biomarker for cervical cancer is the squamous cell carcinoma antigen, which is elevated in $50 \%, 71 \%$, and $82 \%$ of patients with stages I, II, and III-IV disease, respectively [18]. Circulating antibodies [19], CDKN3 mRNA [20], amino acids [21], and lipids [22] also have been studied as potential biomarkers for cervical cancer. However, their diagnostic accuracy and predictive performance remain uncertain.

To the best of our knowledge, this is the first study of its kind to identify, through metabolomic analysis, dysregulated metabolic pathways and potential biomarkers for CINs and cervical cancers. We performed ultra-performance liquid chromatography (UPLC)-MS-based metabolomic profiling in both CINs/cancer patients and normal controls to identify circulating biomarkers of disease progression and validate identified metabolite biomarkers. This global and targeted approach consisting of both discovery and validation steps could be helpful for discovering new biomarkers for risk prediction and early-disease diagnosis.

\section{Results}

\subsection{General Characteristics of Study Participants}

The baseline characteristics of the patients and normal (healthy) controls included in the study are listed in Table 1. The median ages calculated for the normal, CIN1, CIN2/3, and cervical cancer groups were $48,35,39.5$, and 50 years, respectively. Among these groups, $43.5 \%, 54.6 \%, 71.4 \%$, and $78.3 \%$ were positive for HPV infection, respectively. Significant differences $(p<0.05)$ in age, body mass index (BMI), HPV infection, marital status, education, postmenopausal status, pregnancy, and smoking status were observed among the groups; the exception was oral contraceptive use $(p=0.0949)$. Notably, the highest percentages for BMI, HPV, postmenopausal status, and positive pregnancy were recorded in the cervical cancer group. 
Table 1. General characteristics of study subjects.

\begin{tabular}{|c|c|c|c|c|c|c|}
\hline \multicolumn{2}{|c|}{ Variables } & Normal, $n=69$ & CIN1, $n=55$ & CIN2/3, $n=42$ & CX CAN, $n=60$ & $p^{\mathrm{a}}$ \\
\hline \multicolumn{2}{|c|}{ Age (years) } & $48(43,51)$ & $35(31,40)$ & $39.5(33,49)$ & $50(42,51)$ & $<0.0001$ \\
\hline \multicolumn{2}{|c|}{ BMI $\left(\mathrm{Kg} / \mathrm{m}^{2}\right)$} & $21.64(20.5,23.2)$ & $20.6(19.4,21.9)$ & $20.8(19.8,23.4)$ & $23.2(20.6,25.7)$ & 0.0003 \\
\hline \multirow[t]{2}{*}{ HPV } & Positive & $30(43.5)$ & $30(54.6)$ & $30(71.4)$ & $47(78.3)$ & 0.0002 \\
\hline & Single & $4(5.8)$ & $21(38.2)$ & $7(20)$ & $4(6.67)$ & \\
\hline \multirow[t]{3}{*}{ Marital status } & Married & $59(85.5)$ & $29(52.7)$ & $24(68.6)$ & $47(78.3)$ & $<0.0001$ \\
\hline & $\begin{array}{c}\text { Divorce, } \\
\text { bereavement }\end{array}$ & $6(8.7)$ & $5(9.1)$ & $4(11.4)$ & $9(15)$ & \\
\hline & $\leq 9 \mathrm{y}$ & $12(17.4)$ & $2(3.6)$ & $6(17.1)$ & $30(50)$ & \\
\hline \multirow[t]{2}{*}{ Education } & $9-12 y$ & $24(34.8)$ & $19(34.6)$ & $13(37.1)$ & $21(35)$ & $<0.0001$ \\
\hline & $\geq 12 y$ & $33(47.8)$ & $34(61.8)$ & $16(45.7)$ & $9(15.0)$ & \\
\hline Postmenopausal & Yes & $28(40.6)$ & $4(7.3)$ & $8(22.9)$ & $34(56.7)$ & $<0.0001$ \\
\hline \multirow{2}{*}{ Pregnancy } & No & $4(5.8)$ & $21(38.2)$ & $9(25.7)$ & $5(8.3)$ & \\
\hline & Yes & $65(94.2)$ & $34(61.8)$ & $26(74.3)$ & $55(91.7)$ & $<0.0001$ \\
\hline Oral contraceptive & Yes & $11(15.9)$ & $8(14.8)$ & $12(34.3)$ & $9(17.7)$ & 0.0949 \\
\hline Smoking status & $\begin{array}{l}\text { Yes, include } \\
\text { past }\end{array}$ & $8(11.8)$ & $18(32.7)$ & $7(20)$ & $7(11.7)$ & 0.0097 \\
\hline
\end{tabular}

Data are present as median (25\%, 75\%), number (\%). CIN1: cervical intraepithelial neoplasia 1, CIN2/3: cervical intraepithelial neoplasia 2 or 3, CX CAN: cervical cancer. ${ }^{a}$ Kruskal-Wallis test in continuous variables and chi-square test in categorical data.

\subsection{Global Metabolic Profiling of Plasma by UPLC-QTOF-MS}

We performed global profiling of the polar metabolites in the plasma samples to identify altered metabolites in the CIN and cervical cancer patients. During the profiling, the analytical robustness of ultra-performance liquid chromatography-quadrupole-time-of-flight mass spectrometry (UPLC-QTOF-MS) quality control (QC) samples was repeatedly analyzed. In those samples, the reproducibility of the polar metabolite features was evaluated by coefficients of variation (CV); metabolite features showing large variations were removed ( $\mathrm{CV}>20 \%$ ) before conducting the statistical analysis. In the UPLC-QTOF-MS dataset, a total of 1920 metabolite features were detected in the positive mode and 2563 were detected in the negative mode. After annotation, we obtained 52 metabolite features in the positive mode and 40 in the negative mode. To determine whether the metabolic profiles $(\mathrm{CV}<20 \%)$ of the CINs and cervical cancers were different from the normal ones, a multivariate statistical analysis using the principal component analysis (PCA) model was conducted. The score plots obtained from the PCA model are presented in Figure $1 A, B$. The $R^{2} X$ values of the PCA analysis were $>0.6$ for both the positive mode $\left(R^{2} X=0.635\right)$ and the negative mode $\left(R^{2} X=0.666\right)$. We excluded 26 PCA-based-outlier plasma samples (1 CIN1, 17 CIN2/3, 8 cervical cancer) from further analysis. The PCA analysis demonstrated that the four groups were not clearly discriminated; instead, the results had clustered into two groups: A normal/CIN1 group and a CIN2 or 3/cervical cancer group. Importantly, the QC samples were clustered together on the PCA score plot, which indicated the robustness of our polar profiling platform (Figure S1). 
A

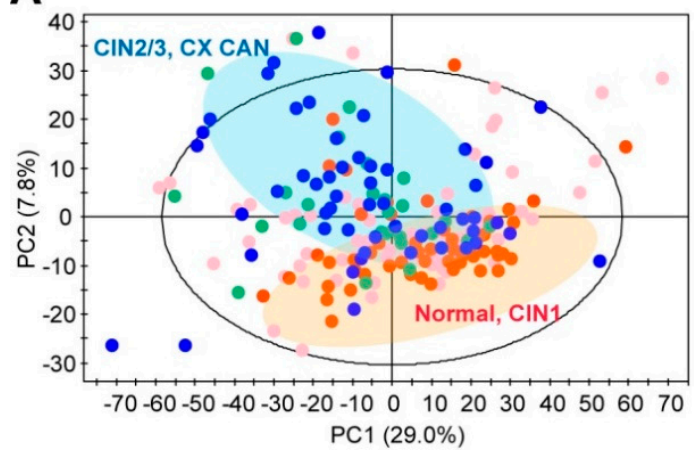

B

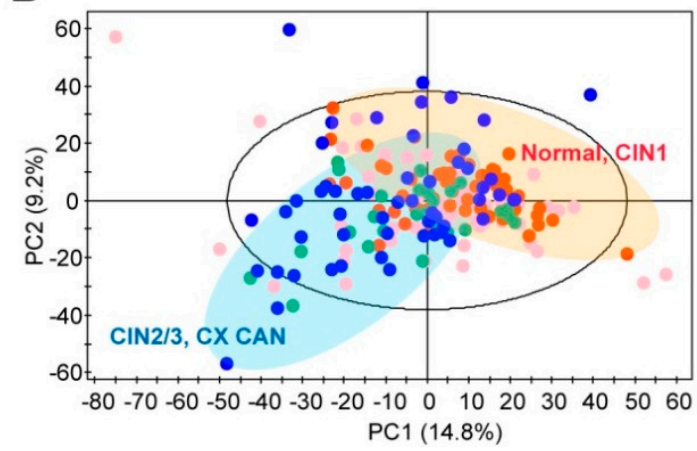

- Normal

- $\mathrm{CIN} 1$

- $\mathrm{CIN} 2 / 3$

- CXCAN

Figure 1. Score plots of principal component analysis (PCA) from metabolite spectra of (A) positive $\left(R^{2} X=0.635\right.$, and $\left.Q^{2}=0.472\right)$ and $(B)$ negative $\left(R^{2} X=0.666\right.$, and $\left.Q^{2}=0.399\right)$ modes of ultra-performance liquid chromatography-quadrupole-time-of-flight mass spectrometry (UPLC-QTOF-MS). Plots of the PCA analysis reveal no clear separations of the four groups, but clustered into two groups: normal/cervical intraepithelial neoplasia (CIN)1 group and CIN2 or 3/cervical cancer group. CIN1: Cervical intraepithelial neoplasia 1, CIN2/3: Cervical intraepithelial neoplasia 2 or 3, CX CAN: Cervical cancer.

\subsection{Differential Mapping of Metabolites in Pathway Analysis}

To explore the metabolic pathways that potentially contribute to cervical cancer progression, we carried out a global metabolic pathway analysis (MetPA website: www.metaboanalyst.ca). By logarithmic transformation, totals of 37, 29, and 33 pathways from significant metabolites were obtained for normal and cervical cancer, CIN1 and cervical cancer, and normal + CIN1 and CIN2/3 + cervical cancer, respectively (false discovery rate (FDR) $p$-value $<0.05$ ), as shown in Figure 2 . Small $p$-values and large pathway-impact values indicated highly influential pathways. Based on the impact values and $p$-values, the pathways of alanine, aspartate and glutamate metabolism, arginine and proline metabolism, taurine and hypotaurine metabolism, and pyruvate metabolism were significantly altered in the cases of normal versus cervical cancer, CIN1 versus cervical cancer, and normal + CIN1 versus CIN2/3 + cervical cancer (Figure S2; FDR $p$-value $<0.05$; impact value $>0.3$ ).
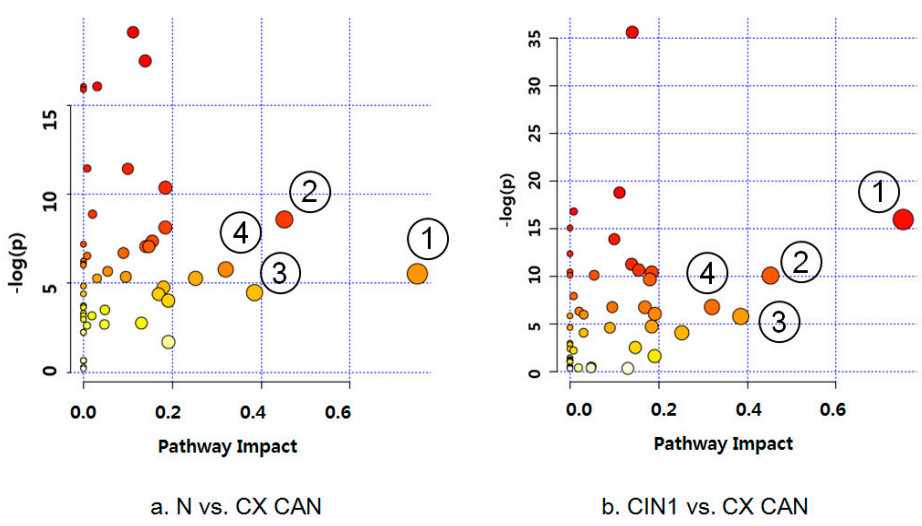

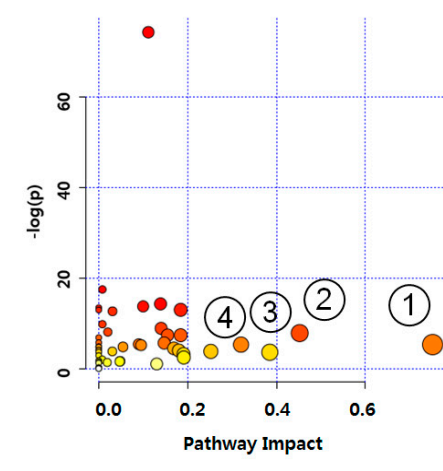

C. $N+$ CIN 1 vs. CIN2/3+CX CAN

Figure 2. Metabolic pathway analysis in cervical carcinogenesis. Global pathway analysis was performed using MetaboAnalyst: (a) Normal and cervical cancer, (b) CIN1 and cervical cancer, (c) normal, CIN1, and CIN2/3, cervical cancer. (1) Alanine, aspartate, and glutamate metabolism, (2) Arginine and proline metabolism, (3) Taurine and hypotaurine metabolism, (4) Pyruvate metabolism. N: Normal, CIN1: Cervical intraepithelial neoplasia 1, CIN2/3: Cervical intraepithelial neoplasia 2 or 3, CX CAN: Cervical cancer. 


\subsection{Altered Metabolites in Patients with CINs and Cervical Cancer}

A total of 28 significantly altered metabolites were identified in the positive mode (19 metabolites) and negative mode (16 metabolites) (Kruskal-Wallis test $p<0.05)$. For global visualization of the significantly altered metabolites, hierarchical cluster analysis (HCA) and area under the curve (AUC) analysis were performed. A dendrogram produced through the HCA revealed three distinct clusters in both the positive and negative modes (Figure S3). Metabolites showing significant trends in plasma levels among the normal, CIN, and cervical cancer groups were identified as potential biomarkers for early detection of cervical cancer. Of these, we chose the top seven metabolites, namely AMP, aspartate, glutamate, hypoxanthine, lactate, proline, and pyroglutamate, on the basis of the HCA clusters and AUC analysis (Table S2).

\subsection{Validation and Diagnostic Performance of Selected Metabolites}

The levels of the selected metabolites in a group of participants comprising 69 normal, 55 CIN1, $42 \mathrm{CIN} 2 / 3$, and 60 cervical cancer patients (Table 1) were measured using UPLC-triple-quadrupole (TQ)-MS and analyzed in the validation phase by Bonfferoni multiple comparisons test. Figure 3 depicts the boxplots of their concentrations in the CIN, cervical cancer, and normal cases. All of the metabolites were up-regulated in CIN and cervical cancer, except for proline metabolite in the CIN1 group. Interestingly, AMP was significantly elevated in the cervical cancer group as compared to CIN and normal, whereas no significant difference was observed for proline between CIN and cervical cancer as compared to normal $(p<0.05)$.
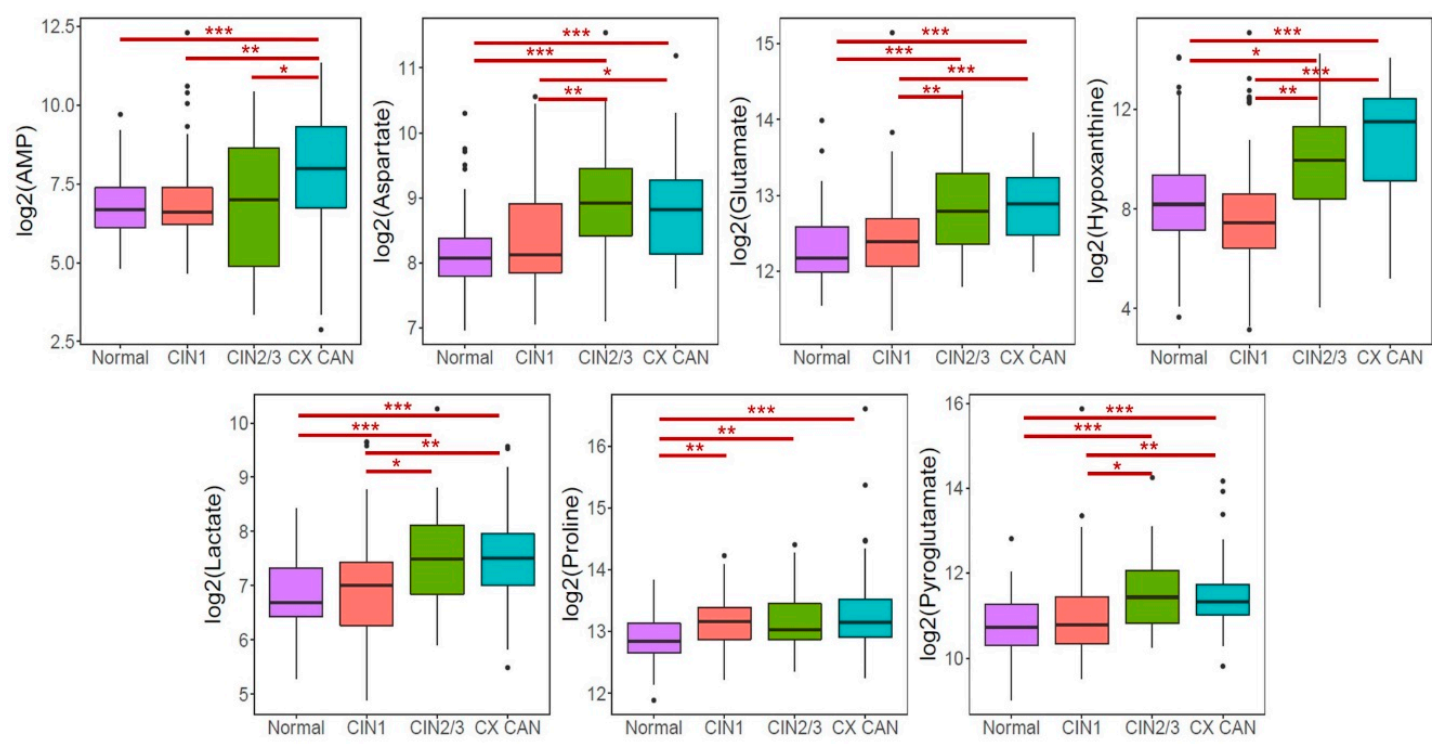

Figure 3. Boxplots of metabolites between normal and cervical cancer. Post-hoc multiple comparisons using Bonfferoni method was performed for significant difference. ${ }^{*} p<0.05,{ }^{* *} p<0.01,{ }^{* * *} p<0.001$. CIN1: Cervical intraepithelial neoplasia 1, CIN2/3: Cervical intraepithelial neoplasia 2 or 3, CX CAN: Cervical cancer.

Table 2 shows the groups' AUC values of targeted metabolites obtained through multiple comparison analysis. The AUC values indicate the diagnostic potentials of the metabolites as unique biomarkers for identification of CINs and cervical cancers. The AUC value (0.80) of aspartate metabolite indicated a clear discrimination between the normal and CIN2/3 groups, whereas, those of glutamate and hypoxanthine metabolites clearly discriminated between normal and cervical cancer and CIN1 and cervical cancer, respectively. We thereafter combined the metabolites into various sets and subjected them to AUC analysis to evaluate their diagnostic performances as combined biomarkers for CINs and cervical cancers. The AUC value of three combined metabolites (aspartate, glutamate, 
and hypoxanthine) was 0.81 between the normal and CIN2/3 group and the normal and cervical cancer group; the AUC values of seven combined metabolites (AMP, aspartate, glutamate, hypoxanthine, lactate, proline, and pyroglutamate) was 0.82 between the normal and CIN2/3 group and 0.83 between the normal and cervical cancer group. 
Table 2. Area under the curves (AUCs) among normal, CINs, and cervical cancers.

\begin{tabular}{|c|c|c|c|c|c|}
\hline Metabolite & $\mathrm{N}$ vs. CIN $2 / 3$ & $\mathrm{~N}$ vs. CX CAN & CIN1 vs. CIN2/3 & CIN1 vs. CX CAN & $\mathrm{N}+\mathrm{CIN} 1$ vs. CIN2/3 + CX CAN \\
\hline AMP & 0.53 & 0.71 & $<0.50$ & 0.68 & 0.62 \\
\hline 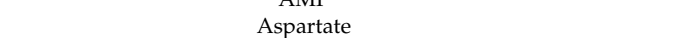 & 0.80 & 0.76 & 0.71 & 0.67 & 0.74 \\
\hline Glutamate & 0.76 & 0.81 & 0.69 & 0.73 & 0.76 \\
\hline Hypoxanthine & 0.68 & 0.77 & 0.72 & 0.80 & 0.74 \\
\hline Lactate & 0.74 & 0.74 & 0.67 & 0.69 & 0.71 \\
\hline Proline & 0.68 & 0.71 & 0.51 & 0.54 & 0.62 \\
\hline Pyroglutamate & 0.72 & 0.74 & 0.68 & 0.69 & 0.71 \\
\hline Aspartate,Glutamate & 0.80 & 0.81 & 0.70 & 0.71 & 0.76 \\
\hline Aspartate,Hypoxanthine & 0.82 & 0.76 & 0.73 & 0.73 & 0.73 \\
\hline Glutamate,Hypoxanthine & 0.76 & 0.81 & 0.67 & 0.76 & 0.76 \\
\hline Aspartate,Glutamate,Hypoxanthine & 0.81 & 0.81 & 0.73 & 0.74 & 0.75 \\
\hline AMP,Aspartate,Glutamate,Hypoxanthine & 0.82 & 0.82 & 0.71 & 0.73 & 0.75 \\
\hline Aspartate,Glutamate,Hypoxanthine,Lactate & 0.80 & 0.80 & 0.73 & 0.73 & 0.76 \\
\hline Aspartate,Glutamate,Hypoxanthine,Proline & 0.82 & 0.81 & 0.72 & 0.72 & 0.75 \\
\hline Aspartate,Glutamate,Hypoxanthine,Pyroglutamate & 0.80 & 0.81 & 0.74 & 0.76 & 0.76 \\
\hline AMP,Aspartate,Glutamate,Hypoxanthine,Lactate & 0.81 & 0.82 & 0.71 & 0.73 & 0.75 \\
\hline AMP,Aspartate,Glutamate,Hypoxanthine,Proline & 0.82 & 0.83 & 0.72 & 0.72 & 0.75 \\
\hline AMP,Aspartate,Glutamate,Hypoxanthine,Pyroglutamate & 0.81 & 0.82 & 0.73 & 0.77 & 0.78 \\
\hline Aspartate, Glutamate,Hypoxanthine,Lactate,Proline & 0.82 & 0.81 & 0.72 & 0.72 & 0.75 \\
\hline Aspartate,Glutamate,Hypoxanthine,Lactate,Pyroglutamate & 0.81 & 0.80 & 0.74 & 0.76 & 0.77 \\
\hline $\begin{array}{l}\text { AMP,Aspartate,Glutamate,Hypoxanthine,Lactate,Proline } \\
\text { AMP }\end{array}$ & 0.82 & 0.83 & 0.71 & 0.72 & 0.75 \\
\hline AMP,Aspartate,Glutamate,Hypoxanthine,Lactate,Pyroglutamate & 0.81 & 0.82 & 0.72 & 0.78 & 0.78 \\
\hline Aspartate,Glutamate,Hypoxanthine,Lactate,Proline,Pyroglutamate & 0.82 & 0.82 & 0.73 & 0.77 & 0.78 \\
\hline AMP,Aspartate,Glutamate,Hypoxanthine,Lactate,Proline,Pyroglutamate & 0.82 & 0.83 & 0.72 & 0.78 & 0.78 \\
\hline
\end{tabular}

AMP: Adenosine monophosphate, N: Normal, CIN1: Cervical intraepithelial neoplasia 1, CIN2/3: Cervical intraepithelial neoplasia 2 or 3, CX CAN: Cervical cancer. 


\subsection{Association of Metabolites with CINs and Cervical Cancer Risk}

Table 3 displays the associations of targeted metabolites with CIN2/3 or cervical cancer risk by multivariate logistic analysis. As can be seen, most of the metabolites were significantly associated with increased risk of developing CIN2/3 and cervical cancer from normal. Aspartate, moreover, was significantly associated with increased risk of developing cervical cancer from normal. Note also that a high level of aspartate was shown to have increased risk of developing CIN2/3 compared with CIN1 (OR, 8.92; CI, 2.38-33.4) and CIN2/3 from normal (OR, 4.31; CI, 1.38-13.5). For glutamate, the odds ratios (ORs) and correspondent confidence intervals (CIs) for the risk of developing CIN2/3 and cervical cancer from normal were 7.99 (2.31-27.6) and 6.31 (1.74-22.9), respectively. Hypoxanthine also showed an increased risk of developing CIN2/3 compared with CIN1 (OR, 7.90; CI, 2.00-31.3). The elevated level of pyroglutamate was associated with higher risks of developing cervical cancer (OR range 2.94-8.02). All of the metabolites at the elevated level showed high risks (OR range 2.12-4.49) of developing CIN2/3 + cervical cancer from normal + CIN1. These results indicated that higher levels of metabolites were significantly associated with increased risks of developing CIN2/3 and cervical cancer. 
Table 3. Associations of targeted metabolites with CIN2/3 and cervical cancer risk.

\begin{tabular}{|c|c|c|c|c|c|c|c|c|c|c|c|c|c|}
\hline \multirow[t]{2}{*}{ Metabolite } & $\mathbf{N}$ & $\operatorname{CIN} 2 / 3$ & $\mathrm{~N}$ vs. CIN2/3 & CX CAN & $\mathrm{N}$ vs. CX CAN & CIN1 & CIN23 & CIN1 vs. CIN2/3 & CX CAN & CIN1 vs. CX CAN & $\mathrm{N}+\mathrm{CIN} 1$ & CIN23 + CX CAN & $\begin{array}{c}\mathrm{N}+\text { CIN1 vs. } \\
\text { CIN23 + CX CAN } \\
\end{array}$ \\
\hline & $n=69$ & $n=42$ & $\begin{array}{c}\mathrm{mOR}(95 \% \\
\mathrm{CI})\end{array}$ & $n=60$ & mOR $(95 \% \mathrm{CI})$ & $n=55$ & $n=42$ & $\operatorname{mOR}(95 \% \mathrm{CI})$ & $n=60$ & $\operatorname{mOR}(95 \% \mathrm{CI})$ & $n=124$ & $n=102$ & mOR $(95 \% \mathrm{CI})$ \\
\hline \multicolumn{14}{|l|}{ AMP } \\
\hline Low & 49.3 & 38.1 & ref & 25 & ref & 40.1 & 35.7 & ref & 25 & ref & 50 & 30.4 & ref \\
\hline High & 50.7 & 61.9 & $2.11(0.79-5.63)$ & 75 & $1.52(0.54-4.28)$ & 50.9 & 64.3 & $4.34(1.29-14.6)$ & 75 & $0.62(0.12-3.28)$ & 50 & 69.6 & $2.34(1.13-4.83)$ \\
\hline \multicolumn{14}{|l|}{ Aspartate } \\
\hline Low & 50.7 & 11.9 & ref & 21.7 & ref & 49.1 & 14.3 & ref & 25 & ref & 50 & 20.6 & ref \\
\hline High & 49.3 & 88.1 & $4.31(1.38-13.5)$ & 78.3 & $1.64(0.59-4.58)$ & 50.9 & 85.7 & $8.92(2.38-33.4)$ & 75 & $0.81(0.15-4.24)$ & 50 & 79.4 & $2.80(1.36-5.78)$ \\
\hline \multicolumn{14}{|l|}{ Glutamate } \\
\hline Low & 50.7 & 11.9 & ref & 8.3 & ref & 50.9 & 28.6 & ref & 20 & ref & 50.8 & 14.7 & ref \\
\hline High & 49.3 & 88.1 & $7.99(2.31-27.6)$ & 91.7 & $6.31(1.74-22.9)$ & 49.1 & 71.4 & $2.68(0.87-8.20)$ & 80 & $0.60(0.10-3.71)$ & 49.2 & 85.3 & $4.49(2.05-9.82)$ \\
\hline \multicolumn{14}{|l|}{ Hypoxanthine } \\
\hline Low & 49.3 & 23.8 & ref & 15 & ref & 50.9 & 14.3 & ref & 8.3 & ref & 50.8 & 17.7 & ref \\
\hline \multirow{2}{*}{\multicolumn{14}{|c|}{ Lactate }} \\
\hline & & & & & & & & & & & & & \\
\hline Low & 50.7 & 14.3 & ref & 13.3 & ref & 50.9 & 33.3 & ref & 25 & ref & 50 & 20.6 & ref \\
\hline High & 49.3 & 85.7 & $5.18(1.74-15.4)$ & 86.7 & $3.93(1.24-12.5)$ & 49.1 & 66.7 & $1.98(0.68-5.78)$ & 75 & $0.96(0.17-5.50)$ & 50 & 79.4 & $2.30(1.13-4.69)$ \\
\hline \multicolumn{14}{|l|}{ Proline } \\
\hline Low & 50.7 & 23.8 & ref & 20 & ref & 50.9 & 54.8 & ref & 53.3 & ref & 50 & 30.4 & ref \\
\hline High & 49.3 & 76.2 & $5.13(1.71-15.4)$ & 80 & $5.23(1.50-18.3)$ & 49.1 & 45.2 & $1.04(0.35-3.09)$ & 46.7 & $0.69(0.12-3.94)$ & 50 & 69.6 & $2.12(1.02-4.43)$ \\
\hline \multicolumn{14}{|l|}{ Pyroglutamate } \\
\hline Low & 50.7 & 23.8 & ref & 10 & ref & 49.1 & 23.8 & ref & 11.7 & ref & 50.8 & 16.7 & ref \\
\hline High & 49.3 & 76.2 & $2.94(1.10-7.84)$ & 90 & $8.02(2.13-30.3)$ & 50.9 & 76.2 & $5.46(1.63-18.4)$ & 88.3 & $4.54(0.65-32.0)$ & 49.2 & 83.3 & $4.14(1.92-8.92)$ \\
\hline
\end{tabular}

mOR: Multivariate odds ratio, CI: Confidence interval, ref: Reference, AMP: Adenosine monophosphate, N: Normal, CIN1: Cervical intraepithelial neoplasia 1, CIN2/3: Cervical intraepithelial neoplasia 2 or 3, CX CAN: Cervical cancer. Adjusted for age, BMI, marital status, education, menopause status, parity, smoking status. 
Table 4. Combined effects of metabolites with human papillomavirus (HPV) status on CIN2/3 and cervical cancer risk.

\begin{tabular}{|c|c|c|c|c|c|c|}
\hline \multirow{2}{*}{ Metabolite } & \multirow{2}{*}{ HPV } & $\mathrm{N}$ vs. CIN2/3 & $\mathrm{N}$ vs. CX CAN & CIN1 vs. CIN2/3 & CIN1 vs. CX CAN & $\mathrm{N}+$ CIN1 vs. CIN2/3 + CX CAN \\
\hline & & mOR $(95 \%$ CI) & mOR $(95 \%$ CI) & mOR $(95 \% \mathrm{CI})$ & mOR $(95 \% \mathrm{CI})$ & mOR $(95 \% \mathrm{CI})$ \\
\hline \multicolumn{7}{|l|}{ AMP } \\
\hline Low & $\mathrm{Neg}$ & ref & ref & ref & ref & ref \\
\hline Low & Pos & $6.56(0.99-43.2)$ & $5.36(0.99-29.0)$ & $5.32(0.79-35.9)$ & $5.31(0.98-28.8)$ & $3.36(1.02-11.1)$ \\
\hline High & $\mathrm{Neg}$ & $1.90(0.27-13.3)$ & $0.87(0.12-6.06)$ & $1.82(0.26-12.8)$ & $0.85(0.12-5.94)$ & $1.24(0.32-4.82)$ \\
\hline High & Pos & $12.19(2.21-67.1)$ & $8.54(1.63-44.8)$ & $12.78(2.31-70.7)$ & $8.46(1.61-44.6)$ & $9.33(2.94-29.6)$ \\
\hline$p$ interaction ${ }^{1}$ & & 0.0022 & 0.0184 & 0.0008 & 0.0184 & $<0.0001$ \\
\hline$p$ trend $^{2}$ & & 0.0065 & 0.0489 & 0.0034 & 0.0513 & 0.0002 \\
\hline \multicolumn{7}{|l|}{ Aspartate } \\
\hline Low & $\mathrm{Neg}$ & ref & ref & ref & ref & ref \\
\hline Low & Pos & $2.52(0.31-20.5)$ & $3.58(0.67-19.2)$ & $3.45(0.47-25.3)$ & $4.67(0.93-23.4)$ & $2.47(0.73-8.34)$ \\
\hline High & $\mathrm{Neg}$ & $1.64(0.24-11.2)$ & $0.46(0.06-3.46)$ & $1.74(0.25-12.0)$ & $0.50(0.07-3.76)$ & $1.08(0.28-4.13)$ \\
\hline High & Pos & $13.93(2.62-74.0)$ & $7.71(1.60-37.1)$ & $13.52(2.55-71.7)$ & $6.97(1.45-33.4)$ & $9.15(2.96-28.3)$ \\
\hline$p$ interaction & & $<0.0001$ & 0.0067 & 0.0002 & 0.0236 & $<0.0001$ \\
\hline$p$ trend & & 0.0009 & 0.041 & 0.0013 & 0.0802 & $<0.0001$ \\
\hline \multicolumn{7}{|l|}{ Glutamate } \\
\hline Low & $\mathrm{Neg}$ & ref & ref & ref & ref & ref \\
\hline Low & Pos & 0.98 (0.11-9.18) & $1.14(0.11-12.3)$ & $3.62(0.69-18.9)$ & $3.28(0.60-18.0)$ & $2.76(0.68-11.3)$ \\
\hline High & $\mathrm{Neg}$ & $1.49(0.20-11.3)$ & $1.33(0.16-10.7)$ & $1.51(0.21-10.6)$ & $0.92(0.12-7.06)$ & $2.29(0.56-9.40)$ \\
\hline High & Pos & $27.18(4.23-175)$ & $24.52(3.61-167)$ & $17.01(3.38-85.6)$ & $14.33(2.64-77.8)$ & $14.85(4.27-51.7)$ \\
\hline$p$ interaction & & $<0.0001$ & $<0.0001$ & 0.0002 & 0.001 & $<0.0001$ \\
\hline$p$ trend & & $<0.0001$ & 0.0005 & 0.0006 & 0.0047 & $<0.0001$ \\
\hline \multicolumn{7}{|l|}{ Hypoxanthine } \\
\hline Low & $\mathrm{Neg}$ & ref & ref & ref & ref & ref \\
\hline Low & Pos & $7.59(0.93-61.9)$ & $3.35(0.45-24.9)$ & $46.45(2.11-999)$ & $10.83(0.73-161)$ & $2.11(0.56-8.04)$ \\
\hline High & Neg & $5.25(0.61-45.3)$ & $1.82(0.24-13.6)$ & $9.76(0.70-135)$ & $1.27(0.18-9.21)$ & $1.58(0.42-6.05)$ \\
\hline High & Pos & $21.89(3.36-142)$ & $14.88(2.57-86.2)$ & $33.48(2.83-396)$ & $8.19(1.44-46.6)$ & $11.18(3.55-35.2)$ \\
\hline$p$ interaction & & 0.0005 & 0.0009 & 0.003 & 0.0076 & $<0.0001$ \\
\hline$p$ trend & & 0.0006 & 0.0023 & 0.0024 & 0.0216 & $<0.0001$ \\
\hline \multicolumn{7}{|l|}{ Lactate } \\
\hline Low & $\mathrm{Neg}$ & ref & ref & ref & ref & ref \\
\hline Low & Pos & $2.33(0.31-17.8)$ & $5.35(0.69-41.7)$ & $10.40(1.80-60.0)$ & $3.30(0.71-15.4)$ & $4.24(1.23-14.6)$ \\
\hline High & $\mathrm{Neg}$ & $1.86(0.27-12.8)$ & $2.79(0.36-21.4)$ & $3.57(0.50-25.3)$ & $0.34(0.03-3.8)$ & $1.63(0.42-6.36)$ \\
\hline High & Pos & $17.99(3.31-97.8)$ & $26.84(3.69-196)$ & $16.54(2.86-95.7)$ & $12.01(2.35-61.3)$ & $9.93(3.05-32.4)$ \\
\hline$p$ interaction & & $<0.0001$ & 0.0006 & 0.0105 & 0.0022 & $<0.0001$ \\
\hline$p$ trend & & 0.0002 & 0.0013 & 0.0058 & 0.0139 & 0.0003 \\
\hline
\end{tabular}


Table 4. Cont.

\begin{tabular}{|c|c|c|c|c|c|c|}
\hline \multirow{2}{*}{ Metabolite } & \multirow{2}{*}{ HPV } & $\mathrm{N}$ vs. CIN2/3 & N vs. CX CAN & CIN1 vs. CIN2/3 & CIN1 vs. CX CAN & $\mathrm{N}+\mathrm{CIN} 1$ vs. CIN2/3 + CX CAN \\
\hline & & mOR $(95 \%$ CI) & mOR $(95 \% \mathrm{CI})$ & mOR (95\% CI) & mOR $(95 \% \mathrm{CI})$ & mOR $(95 \% \mathrm{CI})$ \\
\hline \multicolumn{7}{|l|}{ Proline $^{3}$} \\
\hline Low & $\mathrm{Neg}$ & ref & ref & ref & ref & ref \\
\hline Low & Pos & - & $4.16(0.52-33.5)$ & $6.61(1.53-28.6)$ & $4.83(1.07-21.8)$ & $7.65(1.95-29.9)$ \\
\hline High & Neg & - & $2.79(0.35-22.2)$ & $4.29(0.63-29.2)$ & $1.38(0.18-10.7)$ & $3.10(0.73-13.2)$ \\
\hline High & Pos & - & $34.22(3.96-296)$ & $66.90(8.82-507)$ & $22.04(3.60-135)$ & $13.46(3.51-51.7)$ \\
\hline$p$ interaction & & - & 0.0003 & 0.0004 & 0.0028 & $<.0001$ \\
\hline$p$ trend & & - & 0.0009 & 0.0001 & 0.0029 & $<.0001$ \\
\hline \multicolumn{7}{|l|}{ Pyroglutamate } \\
\hline Low & $\mathrm{Neg}$ & ref & ref & ref & ref & ref \\
\hline Low & Pos & $6.76(0.92-49.9)$ & $3.81(0.39-37.7)$ & $6.90(0.94-50.5)$ & $6.71(0.75-60.2)$ & $4.23(1.07-16.8)$ \\
\hline High & $\mathrm{Neg}$ & $2.68(0.34-21.0)$ & $4.59(0.56-37.4)$ & $2.83(0.36-22.0)$ & $4.76(0.60-37.7)$ & $3.15(0.77-12.9)$ \\
\hline High & Pos & $15.50(2.79-86.0)$ & $27.93(4.03-194)$ & $15.79(2.86-87.1)$ & $22.76(3.47-149)$ & $16.17(4.55-57.5)$ \\
\hline$p$ interaction & & 0.001 & 0.0004 & 0.001 & 0.0013 & $<0.0001$ \\
\hline$p$ trend & & 0.0019 & 0.0005 & 0.0017 & 0.0011 & $<0.0001$ \\
\hline
\end{tabular}

CI: Confidence interval; AMP: Adenosine monophosphate; N: Normal, CIN1: Cervical intraepithelial neoplasia 1, CIN2/3: Cervical intraepithelial neoplasia 2 or 3, CX CAN: Cervical cancer, POS: Positive; NEG: Negative. Adjusted for age, BMI, marital status, education, menopause status, parity, smoking status. ${ }^{1} \mathrm{P}$ is for the interaction provided by the logistic regression for multiplicative terms $(\mathrm{a} \times \mathrm{b}) .{ }^{2} \mathrm{P}$ is for the trend according to the order of combination provided by the logistic regression analysis. ${ }^{3}$ The analysis of proline in $\mathrm{N}$ vs. CIN2/3 groups was not possible because of the small sample size. 


\subsection{Combined Effects of Targeted Metabolites with HPV Status}

Table 4 displays the combined effects of HPV infection with targeted metabolites on the development risks of CIN2/3 and cervical cancer. As expected, high levels of targeted metabolites with positive HPV status showed a significant risk for development of CIN2/3 and cervical cancer. At the elevated level, proline showed the highest risk (OR 66.9; $p$ for trend $=0.0001)$ of developing CIN2/3 from CIN1, followed by hypoxanthine, glutamate, and lactate (OR range 16.54-33.48; $p$ for trend $<0.01$ ). Similarly, proline, pyroglutamate, lactate, and glutamate at the elevated level also demonstrated increased risk for developing cervical cancer from normal (OR range 24.52-34.22; $p$ for trend $<0.0009$ ). These results indicated that the risk of developing CIN2/3 or cervical cancer is significantly higher if patients are positive for HPV infection.

\subsection{Pathway Analysis for Quantitative Metabolites}

Metabolomic pathway analysis was performed for the seven targeted metabolites using the MetPA web-based tool. The highest impact value was obtained for the alanine, aspartate, and glutamate metabolic pathways; these results were consistent with the global pathway analysis (Figures S4 and S5; impact value $>0.4$, FDR $p$-value $<0.05$ ). Metabolic pathways including arginine and proline metabolism, pyruvate metabolism, aminoacyl-tRNA biosynthesis, and D-glutamine and D-glutamate metabolism were statistically related (impact $>0.1$, FDR $p$-value $<0.05$ ).

\section{Discussion}

Alteration in metabolic pathways has been involved in the development of neoplasia and is characterized as a hallmark of cancer [23]. A growing body of evidence shows that malignant transformation requires large amounts of energy to sustain high rates of cell proliferation and energy consumption, which leads to alterations of key metabolic pathways [24,25].

In this study, we used global and targeted metabolomic profiling to identify plasma metabolite biomarkers for non-invasive early detection of cervical cancer and as risk factors for cancer development. Cervical cancer causes changes in body metabolism, and results in alterations in circulating metabolites. Thus, investigation of cervical-cancer-associated metabolic pathways can provide important insights into the different mechanisms adopted during the development and progression of tumors and proposes new methods for its early detection. Our findings showed that 28 metabolites among normal, CINs, and cervical cancers were significantly altered in the global metabolite profiling (discovery phase). These metabolites were successfully mapped to 37 normal and cervical cancer, 29 CIN1 and cervical cancer, and 33 normal + CIN1 and CIN2/3 + cervical cancer pathways. The results obtained from our pathway analysis indicated that the metabolic pathways of alanine, aspartate, and glutamate metabolism, arginine and proline metabolism, taurine and hypotaurine metabolism, and pyruvate metabolism were significantly altered between cancer patients and the normal group. In the validation phase, seven metabolites that were statistically different in cervical carcinogenesis progression were selected as potential biomarkers on the basis of AUC (>0.80) and HCA analyses. Furthermore, HPV, a known causal agent of cervical cancer development, was shown to represent high risk when combined with targeted altered metabolites in the validation phase.

Endogenous and exogenous amino acids are considered to be vital sources of nutrients that are distributed all over the body to contribute to metabolism, gene expression, cell multiplications, and inflammatory reactions. The speedy proliferation and increased metabolism of tumor cells require amino acids for protein and nucleic acids synthesis [26]. It has already been reported that amino acids such as serine, glutamine, aspartic acid, and proline metabolism play an important role in cancer progression and contribute to tumor metabolic reprogramming [27]. The analysis of sera from cancer patients often shows altered amino acid profiles compared with healthy patients [28,29]. Hasim, Aili, Maimaiti, Abudula, and Upur [21] showed that the total serum concentrations of 
aspartate, proline, and glutamate, among others, were gradually decreased from CIN to invasive cancer. In another study, the concentration of L-lysine essential amino acid also was decreased in cervical cancer patients [30]. Ye et al. [31] reported that metabolomics profile of serum samples obtained from cervical cancer patients exhibited reduced levels of most essential and non-essential amino acids, such as isoleucine, valine, tyrosine, and glycine, among others as compared to cervicitis and CIN. Correspondingly, we found altered amino acids profiles in cervical cancer patients compared with normal patients. By contrast, we found elevated levels of plasma aspartate, glutamate, proline, and pyroglutamate in cervical cancer patients compared with those of normal individuals (Figure 3). It is an established fact that metabolism, including that of amino acids, changes not only in cancerous cells, but also in plasma [32,33]. However, there are inconsistencies among altered amino acid profiles of cancer patients $[21,34]$ as well as discrepancies between the reported literature and our present results. Differences among the findings of various studies can be attributed to study design, detection procedures, pre-cancerous lesion status, and sample size. However, findings similar to ours also are available. For example, some studies have reported elevated levels of amino acid profiles in cancerous patients [35,36]. Augmentation of amino acids in the plasma of cancer patients indicates the utilization of amino acids for protein synthesis required for increased cell growth [27]. These results suggest that cancer progression as characterized by the severity of invasion and metastasis is highly associated with plasma amino acid profiles [37]. However, it remains unclear how metabolic changes occurring in cancer patients influence plasma amino-acid profiles systemically.

Lactate metabolite is normally found in elevated levels in tumor hypoxia, a condition in which cells acquire energy through glycolysis instead of oxidative phosphorylation [38]. We found significantly elevated levels of lactate in CIN and cervical cancer cases compared with normal individuals. In cervical cancer patients relative to CIN2/3 patients, the lactate metabolites levels were slightly reduced. Several studies have reported similar findings of elevated levels of lactate in other types of cancer [39,40]. In contrast to our findings, Hasim et al. [41] reported reduced levels of lactate in CIN and cervical cancer as compared with normal individuals. The discrepancy in these results could have been due to differences in study design and detection methods.

Notably, we observed that metabolites from the purine metabolic pathway, AMP, and hypoxanthine were associated with cervical cancer risk. During progression from normal to CIN1, the level of hypoxanthine reduced and then increased in CIN2/3 and cervical cancer when compared to normal individuals. Hypoxanthine has been described as a protective agent against hypoxic injury and cytotoxic compounds in normal and cancerous cells [42]. It is oxidized to xanthine by xanthine oxidase (a form of xanthine oxidoreductase), whereas uric acid is the end product of their degradation [43]. Previous studies have suggested that uric acid might contribute to cancer risk, recurrence, and mortality [44], which conclusions point to the importance of this metabolic pathway in influencing cancer progression and outcomes. AMP plays important roles in the regulation of cell proliferation and in acting as an intermediate in purine metabolism. Elevated levels of AMP can be attributed to increased conversion of adenosine to AMP by adenosine kinase enzymes during cell proliferation [45]. In agreement with our findings, Satoh et al. [46] applied a multiomics approach to paired normal and tumor tissue obtained from 275 colorectal cancer patients and found that almost all metabolic genes of the de novo purine/pyrimidine synthesis pathway were up-regulated. Consistent with our findings, Sahu et al. [47] performed metabolomic analysis in urothelial carcinoma and found elevated levels of both purine and pyrimidine metabolites.

Although a cancerous tumor is limited to a certain organ, changes in metabolite levels are influenced by their metabolism in, and excretion from, several organs of the body. Therefore, the blood plasma of cancer patients contains almost all of the metabolite information related to pathogenic alterations triggered by disease, which data reveal are abnormal alterations at the gene expression and regulation levels as well as aberrations in the functions of multiple organs and tissues. However, as already noted in these pages, it remains unclear how changes in metabolic profiles 
occurring in pre-cancerous and cancerous patients systematically affect plasma metabolites, including amino acid.

Previous epidemiological studies have shown associations between various metabolites and cervical cancer risk; for example, levels of serum triglyceride and systolic blood pressure were positively associated with cervical cancer risk [48-51]. Walker, Burrell, Flatley, and Powers [8] performed metabolomic analysis and suggested that metabolite profiling could be used to rapidly identify women at increased risk of cervical cancer. Likewise, we also found substantial associations between targeted metabolites and CIN2/3 or cervical cancer risk (Table 3). These results indicate an increased risk of developing CIN2/3 and cervical cancer from a normal group having elevated levels of these metabolites in plasma.

Nearly all cases of cervical cancer can be attributed to HPV infection [52]. We have found $78.3 \%$ HPV positive cases for cervical cancer group (Table 1), and the results are in agreement with Lei et al. [53], who studied HPV infection in cervical cancer in a nationwide cohort 2002-2011 and found $80.6 \%$ were positive for HPV infection. In another study, Hooi et al. [54] investigated the prevalence of HPV genotypes in cervical cancers and CIN in Curaçao and found $88.5 \%$ cases were HPV positive. However, HPV status in a cervical tumor may be dependent on various factors such as type of test performed, sample quality, laboratory conditions, and sample size. HPV plays an important role in the progression of precursor lesions and cervical cancers. HPV is essential along with cofactors that aid viral persistence and progression [55]. We have discovered in our current study that HPV infection combined with elevate levels of metabolites substantially increased the risk of cancer progression. Metabolites such as aspartate, glutamate, hypoxanthine, and proline showed an increased risk for the development of CIN2/3 and cervical cancer from normal with highest risk was observed for proline (OR, 66.90; CI, 8.82-507).

Although our approach to establish a screening strategy for cervical cancer and precancerous lesions, plasma metabolomic analysis, and the search for unique metabolic pathways is novel and promising, there were some limitations to this study. First, all of the samples collected were non-fasting ones, which, in fact, potentially increased variability among our subjects. Second, the sample size was small, which could have affected the robustness of our statistical analysis. Third, we performed UPLC-QTOF-MS analysis, which might not be an appropriate screening tool for large populations, due to its high cost in developing countries. Fourth, personal and demographic factors such as sex, age, BMI, diet, and smoking status might influence the metabolomic landscape. Further study is needed to optimize and validate cost-effective methods for detection of plasma metabolic biomarkers. Additionally, careful matching of known risk factors and adjustment of covariates in statistical analysis could be considered in order to avoid false positivity. Nevertheless, global profiling of plasma metabolites with targeted validation and comprehensive collection of epidemiological information within a proper hospital setup could offer important resources for identifying unique metabolic biomarkers suitable for clinical application.

\section{Materials and Methods}

\subsection{Study Population and Sample Collection}

This study included subjects aged 18 to 65 years who had participated in the Korean Prospective Study of the Transition of Human Papillomavirus into Cervical Carcinoma since 2006. These women were randomly selected from the six gynecology and oncology departments located in South Korean university hospitals. Details on the study's design criteria are available in our previous paper [56]. All of the patients had been diagnosed with CINs or cervical cancers histologically. Blood samples were collected from the participants in a non-fasting state and centrifuged at $3000 \mathrm{rpm}$ for $20 \mathrm{~min}$ at $4{ }^{\circ} \mathrm{C}$; the obtained plasma was stored at $-80{ }^{\circ} \mathrm{C}$ until further analysis. A total of 205 samples (70 normal, 54 CIN1, 27 CIN2/3, 54 cervical cancer) were selected for positive-mode global analysis, and 202 (67 normal, 54 CIN1, 27 CIN2/3, 54 cervical cancer) for negative-mode global analysis. 
Additionally, a total of 226 plasma samples ( 69 normal, 55 CIN1, 42 CIN2/3, 60 cervical cancer) were selected for targeted analysis. According to the requirements of the National Cancer Center (NCC)'s Institutional Review Board, written consent was obtained from all of the participants. This study was approved by the NCC's Institutional Ethics Committee (IRB No. NCC2016-0147).

\subsection{Global Metabolite Profiling Using UPLC-QTOF-MS}

A UPLC-QTOF-MS-based platform was used to analyze polar metabolites from chloroform/methanol extract. Briefly, plasma samples $(50 \mu \mathrm{L})$ were extracted using a $500 \mu \mathrm{L}$ chloroform:methanol $(2: 1, \mathrm{v} / \mathrm{v})$ solution and $100 \mu \mathrm{L}$ water. The aqueous supernatant was vacuum dried and reconstituted into $250 \mu \mathrm{L}$ water:acetonitrile $(4: 1, \mathrm{v} / \mathrm{v})$ solution. Finally, $5 \mu \mathrm{L}$ solution was injected into the UPLC-QTOF-MS system. The UPLC-QTOF-MS analysis was performed using the ACQUITY UPLC system (Waters, Milford, MA, USA) coupled with a triple TOFTM 5600 mass spectrometer equipped with an electrospray ionization (ESI) source (AB Sciex, Concord, ON, Canada). Chromatographic separation was carried out at $40{ }^{\circ} \mathrm{C}$ on an Acquity UPLC HSS T3 column $(2.1 \mathrm{~mm} \times 100 \mathrm{~mm}, 1.7 \mu \mathrm{m}$; Waters $)$ with a binary gradient at a flow rate of $0.4 \mathrm{~mL} / \mathrm{min}$. The mobile phases consisted of $0.1 \%$ formic acid in water (solvent $\mathrm{A}$ ) and $0.1 \%$ formic acid in acetonitrile (solvent $B$ ). The steps of the gradient profile used to equilibrate the initial gradient for subsequent runs were as follows: $1 \% \mathrm{~B}, 1 \%-10 \% \mathrm{~B}$ from $0-3 \mathrm{~min}, 10 \%-30 \%$ B from $3-5 \mathrm{~min}, 30 \%-50 \%$ B from 5-10 min, 50\%-70\% B from 10-13 min, 70\%-90\% B from 13-15 min, 90\%-1\% B from $15-18 \mathrm{~min}$, $1 \%$ B from $18-20 \mathrm{~min}$.

The mass spectrometer was operated in both the positive and negative ion modes, and data were acquired in the 50-1000 m/z mass range. Total ion chromatograms were acquired according to the following operation parameters: Capillary voltages of $+5500 \mathrm{~V}$ and $-4500 \mathrm{~V}$ for the positive and negative modes, a nebulizer pressure of $60 \mathrm{psi}$, a drying gas pressure of $60 \mathrm{psi}$, a curtain gas pressure of $30 \mathrm{psi}$, a source temperature of $500{ }^{\circ} \mathrm{C}$, a declustering potential of $\pm 90 \mathrm{eV}$, a collision energy of $\pm 10 \mathrm{eV}$ for single MS, and a collision energy of $\pm 35 \mathrm{eV}$ for MS/MS. Data from the MS/MS analyses were acquired by automatic fragmentation, in which process the five most intense mass peaks were fragmented. Mass accuracy was maintained by use of an automated calibrant delivery system interfaced with the second inlet of the DuoSpray source. Equal amounts of all of the samples were pooled to generate QC samples, which were analyzed prior to sample acquisition and after every 15 samples in order to monitor the stability and reproducibility of the analytical system.

\subsection{Metabolite Quantification Using UPLC-TQ-MS}

Modern TQ-MS provides the ability to detect and quantify a large number of metabolites. The present quantification of targeted metabolites was performed on Agilent 1290 Infinity II LC and Agilent 6495 Triple Quadrupole MS systems equipped with an Agilent Jet Stream ESI source (Agilent Technologies, Palo Alto, CA, USA). MassHunter Workstation (Ver B.06.00, Agilent Technologies) software was used for data acquisition and analysis. Chromatographic separation was performed using a Scherzo SM-C18 column ( $2 \mathrm{~mm} \times 100 \mathrm{~mm}, 3 \mu \mathrm{m}$; Imtakt, Kyoto, Japan). The flow rate and injection volume were set at $0.35 \mathrm{~mL} / \mathrm{min}$ and $1 \mu \mathrm{L}$, respectively. Mobile phase A consisted of $0.1 \%$ formic acid in water, and phase B consisted of $0.1 \%$ formic acid in methanol. The linear gradient used for elution and equilibration of the initial gradient for subsequent runs was as follows: $5 \% \mathrm{~B}$ from 0-3 min, 5-90\% B from 3-10 min, 90\% B from 10-12 min, 5-95\% B from 12-13 min, 5\% B from 13-15 min. Quantification was performed in the multiple reaction monitoring (MRM) mode, and the optimal conditions for each metabolite were determined by flow injection of individual standards $(100 \mathrm{ng} / \mathrm{mL}$ in $20 \%$ methanol) into the ESI source in the positive and negative ion modes. ${ }^{13} \mathrm{C}_{6}$-Phenylalanine was used as an internal standard. The compound retention times and MRM transitions are summarized in Table S1. 


\subsection{Statistical Analysis}

A multivariate statistical analysis was performed using SIMCA-P+ version 12.0 (Umetrics, Umeå, Sweden). PCA using an unsupervised method was applied to obtain an overview of the metabolic data. All of the metabolite variables obtained from the UPLC-QTOF-MS datasets were scaled to unit-variances prior to conducting the PCA. The model validity was evaluated from model parameters $R^{2}$ and $Q^{2}$, which provide information for the interpretability and predictability, respectively, of the model. The differences between groups were compared using Kruskal-Wallis test for continuous variables and chi-square test for categorical variables. The metabolites obtained from global analysis were subjected to Kruskal-Wallis analysis, HCA, and AUC analysis [57,58]. Afterwards, seven metabolites were selected on the basis of the HCA and AUC values. Post-hoc multiple comparisons using the Bonfferoni method were applied to identify significant inter-group differences in the targeted metabolites [59]. An AUC analysis also was performed on the targeted metabolites. Metabolite enrichment was log transformed before being subjected to pathway analysis in MetaboAnalyst version 4.0 (Xia Lab, McGill University, Montreal, QC, Canada) [60]. Unconditional logistic regression was conducted to estimate the ORs and the correspondent CIs $(95 \%)$, which were adjusted for age, BMI, marital status, education, menopause status, pregnancy, and smoking status. To categorize the metabolites, the median value in the normal group was calculated and used as the cutoff point for dichotomization into high- and low-risk groups. The combined effects of HPV status with metabolites were calculated using unconditional logistic regression analysis, and the estimated ORs and CIs (95\%) after the variables were log2-transformed. All of the statistical tests were 2 -sided, with the significance set at $p<0.05$. All of the other statistical analyses and visualizations were performed using the ggplot2 packages in the $\mathrm{R}$ platform $[58,61]$. The overall scheme of the metabolomic analysis for the discovery of novel biomarkers for cervical carcinogenesis is presented in Figure 4.

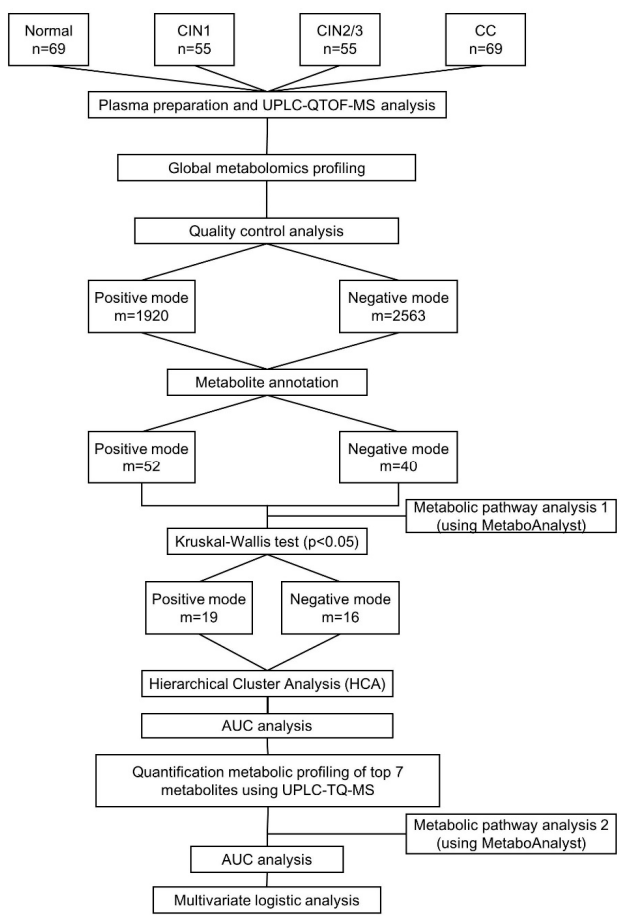

Figure 4. Schematic flow chart of metabolomic analysis in present study. UPLC-QTOF-MS: Ultra-performance liquid chromatography-quadrupole time-of-flight mass spectrometry; UPLC-TQ-MS: Ultra-performance lipid chromatography-triple quadruple mass spectrometry; AUC: Area under the curve; HCA: Hierarchical cluster analysis. 


\section{Conclusions}

The use of metabolomics for detection of cervical cancer biomarkers has been shown by previous studies as well as the present one to be quite promising. The results obtained in the current study showed that 28 metabolites were significantly altered among normal controls, CINs, and cervical cancers in the global profiling phase. We determined that analysis of circulating AMP, aspartate, glutamate, hypoxanthine, lactate, proline, and pyroglutamate levels have the potential to distinguish patients with CINs and cervical cancer from normal (healthy) patients. The risk of developing cervical cancer was high in cases where the metabolite levels were elevated. The risk of developing precancerous lesions and invasive cancer was even higher where patients had elevated levels of metabolites and showed positivity for HPV infection. Further studies are necessary in order to fully understand the association of metabolic changes with cervical cancer progression and their diagnostic potentials.

Supplementary Materials: The following are available online at http:/ / www.mdpi.com/2072-6694/11/4/511/s1, Figure S1: PCA score plots with QC clusters, Figure S2: Construction of the altered metabolism pathways using MetPA, Figure S3: HCA results (a. positive, b. negative mode), Figure S4: Construction of altered metabolic pathways using MetPA analysis for targeted metabolites, Figure S5: Alanine, aspartate, and glutamate metabolism (C00049: Asparate, C00025: Glutamate). Table S1: Retention times and multiple reaction monitoring transitions of plasma metabolites quantified by UPLC-TQ-MS, Table S2: AUC values of significantly altered metabolites $(p$ value $<0.05)$ in given HCA clusters.

Author Contributions: All of the authors contributed significantly to this work. M.K.K., M.K., G.-S.H., and S.-s.S. designed the study; S.-s.S. enrolled the subjects; G.-S.H. supervised metabolomic research; M.N. carried out metabolomic experiments and analyzed the data. S.J. and J.S.H. contributed to the experimental work; M.K. analyzed the results; I.K. drafted the paper. All of the authors read and approved the final manuscript.

Funding: The current work is supported by the National Cancer Center (NCC), South Korea (grant Nos. NCC-1610210 and NCC-1810920). This study was supported by the National Research Foundation (NRF) of Korea (No. 2017M3A9C4065961 and NRF-2017M3A9D5A01052449) and the Korea Basic Science Institute (T39623 and T39720).

Conflicts of Interest: The authors declare no conflict of interest.

\section{Abbreviations}

$\begin{array}{ll}\text { AMP } & \text { Adenosine monophosphate } \\ \text { AUC } & \text { Area under the curve } \\ \text { BMI } & \text { Body mass index } \\ \text { CI } & \text { Confidence interval } \\ \text { CIN } & \text { Cervical intraepithelial neoplasia } \\ \text { CV } & \text { Coefficients of variation } \\ \text { FDR } & \text { False discovery rate } \\ \text { HCA } & \text { Hierarchical cluster analysis } \\ \text { HPV } & \text { Human papillomavirus } \\ \text { OR } & \text { Odds ratio } \\ \text { PCA } & \text { Principal component analysis } \\ \text { QC } & \text { Quality control } \\ \text { UPLC-QTOF-MS } & \text { Ultra-performance liquid chromatography-quadrupole-time-of-flight mass spectrometry } \\ \text { UPLC-TQ-MS } & \text { Ultra-performance liquid chromatography-triple-quadrupole mass spectrometry }\end{array}$

\section{References}

1. Yang, X.; Da, M.; Zhang, W.; Qi, Q.; Zhang, C.; Han, S. Role of Lactobacillus in cervical cancer. Cancer Manag. Res. 2018, 10, 1219. [CrossRef]

2. Fitzmaurice, C.; Dicker, D.; Pain, A.; Hamavid, H.; Moradi-Lakeh, M.; MacIntyre, M.F.; Allen, C.; Hansen, G.; Woodbrook, R.; Wolfe, C.; et al. The Global Burden of Cancer 2013. JAMA Oncol. 2015, 1, 505-527. [CrossRef]

3. Seo, S.-S.; Oh, H.Y.; Lee, J.-K.; Kong, J.-S.; Lee, D.O.; Kim, M.K. Combined effect of diet and cervical microbiome on the risk of cervical intraepithelial neoplasia. Clin. Nutr. 2016, 35, 1434-1441. [CrossRef] 
4. Suehiro, T.T.; Malaguti, N.; Damke, E.; Uchimura, N.S.; Gimenes, F.; Souza, R.P.; da Silva, V.R.S.; Consolaro, M.E.L. Association of human papillomavirus and bacterial vaginosis with increased risk of high-grade squamous intraepithelial cervical lesions. Int. J. Gynecol. Cancer 2019, 29, 242-249. [CrossRef]

5. Yang, J.; Nolte, F.S.; Chajewski, O.S.; Lindsey, K.G.; Houser, P.M.; Pellicier, J.; Wang, Q.; Ehsani, L. Cytology and high risk HPV testing in cervical cancer screening program: Outcome of 3-year follow-up in an academic institute. Diagn. Cytopathol. 2018, 46, 22-27. [CrossRef]

6. Chase, D.; Goulder, A.; Zenhausern, F.; Monk, B.; Herbst-Kralovetz, M. The vaginal and gastrointestinal microbiomes in gynecologic cancers: A review of applications in etiology, symptoms and treatment. Gynecol. Oncol. 2015, 138, 190-200. [CrossRef]

7. Shi, P.; Zhang, L.; Ye, N. Serum metabolomic analysis of cervical cancer patients by gas chromatography-mass spectrometry. Asian J. Chem. 2015, 27, 547-551.

8. Walker, H.; Burrell, M.; Flatley, J.; Powers, H. A metabolite profiling method for diagnosis of precancerous cervical lesions and HPV persistence. Bioanalysis 2017, 9, 601-608. [CrossRef] [PubMed]

9. Nicholson, J.K.; Lindon, J.C. Systems biology: Metabonomics. Nature 2008, 455, 1054-1056. [CrossRef]

10. Khatami, F.; Payab, M.; Sarvari, M.; Gilany, K.; Larijani, B.; Arjmand, B.; Tavangar, S.M. Oncometabolites as biomarkers in thyroid cancer: A systematic review. Cancer Manag. Res. 2019, 11, 1829-1841. [CrossRef]

11. Tebani, A.; Abily-Donval, L.; Afonso, C.; Marret, S.; Bekri, S. Clinical metabolomics: The new metabolic window for inborn errors of metabolism investigations in the post-genomic era. Int. J. Mol. Sci. 2016, 17, 1167. [CrossRef] [PubMed]

12. Wishart, D.S. Quantitative metabolomics using NMR. Trends Anal. Chem 2008, 27, 228-237. [CrossRef]

13. Dettmer, K.; Aronov, P.A.; Hammock, B.D. Mass spectrometry-based metabolomics. Mass Spectrom. Rev. 2007, 26, 51-78. [CrossRef] [PubMed]

14. López-López, Á.; López-Gonzálvez, Á.; Clive-Baker, T.; Barbas, C. A review of validated biomarkers obtained through metabolomics. Expert Rev. Mol. Diagn. 2018, 18, 557-575. [CrossRef] [PubMed]

15. Zhang, F.; Zhang, Y.; Zhao, W.; Deng, K.; Wang, Z.; Yang, C.; Ma, L.; Openkova, M.S.; Hou, Y.; Li, K. Metabolomics for biomarker discovery in the diagnosis, prognosis, survival and recurrence of colorectal cancer: A systematic review. Oncotarget 2017, 8, 35460-35472. [CrossRef]

16. McCartney, A.; Vignoli, A.; Biganzoli, L.; Love, R.; Tenori, L.; Luchinat, C.; Di Leo, A. Metabolomics in breast cancer: A decade in review. Cancer Treat. Rev. 2018, 67, 88-96. [CrossRef]

17. Huang, S.; Chong, N.; Lewis, N.E.; Jia, W.; Xie, G.; Garmire, L.X. Novel personalized pathway-based metabolomics models reveal key metabolic pathways for breast cancer diagnosis. Genome Med. 2016, 8, 34. [CrossRef]

18. Lehtovirta, P.; Viinikka, L.; Ylikorkala, O. Comparison between squamous cell carcinoma-associated antigen and CA-125 in patients with carcinoma of the cervix. Gynecol. Oncol. 1990, 37, 276-278. [CrossRef]

19. Jin, Y.; Kim, S.C.; Kim, H.J.; Ju, W.; Kim, Y.H.; Kim, H.-J. A lectin-based diagnostic system using circulating antibodies to detect cervical intraepithelial neoplasia and cervical cancer. Glycobiology 2015, 26, 100-107. [CrossRef]

20. Barrón, E.V.; Roman-Bassaure, E.; Sánchez-Sandoval, A.L.; Espinosa, A.M.; Guardado-Estrada, M.; Medina, I.; Juárez, E.; Alfaro, A.; Bermúdez, M.; Zamora, R. CDKN3 mRNA as a biomarker for survival and therapeutic target in cervical cancer. PLoS ONE 2015, 10, e0137397. [CrossRef]

21. Hasim, A.; Aili, A.; Maimaiti, A.; Abudula, A.; Upur, H. Plasma-free amino acid profiling of cervical cancer and cervical intraepithelial neoplasia patients and its application for early detection. Mol. Biol. Rep. 2013, 40, 5853-5859. [CrossRef]

22. Yin, M.-z.; Tan, S.; Li, X.; Hou, Y.; Cao, G.; Li, K.; Kou, J.; Lou, G. Identification of phosphatidylcholine and lysophosphatidylcholine as novel biomarkers for cervical cancers in a prospective cohort study. Tumor Biol. 2016, 37, 5485-5492. [CrossRef]

23. Hanahan, D.; Weinberg, R.A. Hallmarks of cancer: The next generation. Cell 2011, 144, 646-674. [CrossRef]

24. Lucarelli, G.; Galleggiante, V.; Rutigliano, M.; Sanguedolce, F.; Cagiano, S.; Bufo, P.; Lastilla, G.; Maiorano, E.; Ribatti, D.; Giglio, A.; et al. Metabolomic profile of glycolysis and the pentose phosphate pathway identifies the central role of glucose-6-phosphate dehydrogenase in clear cell-renal cell carcinoma. Oncotarget 2015, 6, 13371-13386. [CrossRef]

25. Dang, C.V. Links between metabolism and cancer. Genes Dev. 2012, 26, 877-890. [CrossRef]

26. Ward, P.S.; Thompson, C.B. Signaling in control of cell growth and metabolism. Cold Spring Harb. Perspect. Biol. 2012, 4, a006783. [CrossRef] 
27. Liu, W.; Hancock, C.N.; Fischer, J.W.; Harman, M.; Phang, J.M. Proline biosynthesis augments tumor cell growth and aerobic glycolysis: Involvement of pyridine nucleotides. Sci. Rep. 2015, 5, 17206. [CrossRef]

28. Vissers, Y.L.; Dejong, C.H.; Luiking, Y.C.; Fearon, K.C.; von Meyenfeldt, M.F.; Deutz, N.E. Plasma arginine concentrations are reduced in cancer patients: Evidence for arginine deficiency? Am. J. Clin. Nut. 2005, 81, 1142-1146. [CrossRef]

29. Cascino, A.; Muscaritoli, M.; Cangiano, C.; Conversano, L.; Laviano, A.; Ariemma, S.; Meguid, M.; Rossi, F.F. Plasma amino acid imbalance in patients with lung and breast cancer. Anticancer Res. 1995, 15, 507-510.

30. Yang, K.; Xia, B.; Wang, W.; Cheng, J.; Yin, M.; Xie, H.; Li, J.; Ma, L.; Yang, C.; Li, A. A comprehensive analysis of metabolomics and transcriptomics in cervical cancer. Sci. Rep. 2017, 7, 43353. [CrossRef]

31. Ye, N.; Liu, C.; Shi, P. Metabolomics analysis of cervical cancer, cervical intraepithelial neoplasia and chronic cervicitis by 1H NMR spectroscopy. Eur. J. Gynaecol. Oncol 2015, 36, 174-180.

32. Zhang, Q.; Takahashi, M.; Noguchi, Y.; Sugimoto, T.; Kimura, T.; Okumura, A.; Ishikawa, T.; Kakumu, S. Plasma amino acid profiles applied for diagnosis of advanced liver fibrosis in patients with chronic hepatitis C infection. Hepatol. Res. 2006, 34, 170-177. [CrossRef]

33. Maeda, J.; Higashiyama, M.; Imaizumi, A.; Nakayama, T.; Yamamoto, H.; Daimon, T.; Yamakado, M.; Imamura, F.; Kodama, K. Possibility of multivariate function composed of plasma amino acid profiles as a novel screening index for non-small cell lung cancer: A case control study. BMC Cancer 2010, 10, 690. [CrossRef]

34. Mustafa, A.; Gupta, S.; Hudes, G.R.; Egleston, B.L.; Uzzo, R.G.; Kruger, W.D. Serum amino acid levels as a biomarker for renal cell carcinoma. J. Urol. 2011, 186, 1206-1212. [CrossRef]

35. Mayers, J.R.; Wu, C.; Clish, C.B.; Kraft, P.; Torrence, M.E.; Fiske, B.P.; Yuan, C.; Bao, Y.; Townsend, M.K.; Tworoger, S.S. Elevation of circulating branched-chain amino acids is an early event in human pancreatic adenocarcinoma development. Nat. Med. 2014, 20, 1193-1198. [CrossRef]

36. Kratochvilova, M.; Raudenska, M.; Heger, Z.; Richtera, L.; Cernei, N.; Adam, V.; Babula, P.; Novakova, M.; Masarik, M.; Gumulec, J. Amino acid profiling of zinc resistant prostate cancer cell lines: Associations with cancer progression. Prostate 2017, 77, 604-616. [CrossRef]

37. Fan, J.; Hong, J.; Hu, J.-D.; Chen, J.-L. Ion chromatography based urine amino acid profiling applied for diagnosis of gastric cancer. Gastroenterol. Res. Pract. 2012, 2012, 474907. [CrossRef]

38. Morvan, D.; Demidem, A. Metabolomics by proton nuclear magnetic resonance spectroscopy of the response to chloroethylnitrosourea reveals drug efficacy and tumor adaptive metabolic pathways. Cancer Res. 2007, 67, 2150-2159. [CrossRef]

39. Odunsi, K.; Wollman, R.M.; Ambrosone, C.B.; Hutson, A.; McCann, S.E.; Tammela, J.; Geisler, J.P.; Miller, G.; Sellers, T.; Cliby, W.; et al. Detection of epithelial ovarian cancer using ${ }^{1} \mathrm{H}-\mathrm{NMR}-$ based metabonomics. Int. J. Cancer 2005, 113, 782-788. [CrossRef]

40. Fan, T.W.-M. Metabolite profiling by one-and two-dimensional NMR analysis of complex mixtures. Prog. Nucl. Magn. Reson. Spectrosc. 1996, 28, 161-219. [CrossRef]

41. Hasim, A.; Ali, M.; Mamtimin, B.; Ma, J.Q.; Li, Q.-Z.; Abudula, A. Metabonomic signature analysis of cervical carcinoma and precancerous lesions in women by ${ }^{1} \mathrm{H}$ NMR spectroscopy. Exp. Ther. Med. 2012, 3, 945-951. [CrossRef]

42. Obajimi, O.; Melera, P.W. The depletion of cellular ATP by AG2034 mediates cell death or cytostasis in a hypoxanthine-dependent manner in human prostate cancer cells. Cancer Chem. Pharmacol. 2008, 62, 215-226. [CrossRef]

43. Hille, R. Molybdenum-containing hydroxylases. Arch. Biochem. Biophys. 2005, 433, 107-116. [CrossRef] [PubMed]

44. Fini, M.A.; Elias, A.; Johnson, R.J.; Wright, R.M. Contribution of uric acid to cancer risk, recurrence, and mortality. Clin. Trans. Med. 2012, 1, 16. [CrossRef]

45. Spychala, J. Tumor-promoting functions of adenosine. Pharmacol. Ther. 2000, 87, 161-173. [CrossRef]

46. Satoh, K.; Yachida, S.; Sugimoto, M.; Oshima, M.; Nakagawa, T.; Akamoto, S.; Tabata, S.; Saitoh, K.; Kato, K.; Sato, S.; et al. Global metabolic reprogramming of colorectal cancer occurs at adenoma stage and is induced by MYC. Proc. Natl. Acad. Sci. USA 2017, 114, E7697-E7706. [CrossRef]

47. Sahu, D.; Lotan, Y.; Wittmann, B.; Neri, B.; Hansel, D.E. Metabolomics analysis reveals distinct profiles of nonmuscle-invasive and muscle-invasive bladder cancer. Cancer Med. 2017, 6, 2106-2120. [CrossRef]

48. Ulmer, H.; Borena, W.; Rapp, K.; Klenk, J.; Strasak, A.; Diem, G.; Concin, H.; Nagel, G. Serum triglyceride concentrations and cancer risk in a large cohort study in Austria. Br. J. Cancer 2009, 101, 1202-1206. [CrossRef] 
49. Parazzini, F.; La Vecchia, C.; Negri, E.; Fasoli, M.; Cecchetti, G. Risk factors for adenocarcinoma of the cervix: A case-control study. Br. J. Cancer 1988, 57, 201-204. [CrossRef]

50. Ursin, G.; Pike, M.C.; Preston-Martin, S.; d'Ablaing, G.; Peters, R.K. Sexual, reproductive, and other risk factors for adenocarcinoma of the cervix: Results from a population-based case-control study (California, United States). Cancer Causes Control 1996, 7, 391-401. [CrossRef]

51. Ulmer, H.; Bjørge, T.; Concin, H.; Lukanova, A.; Manjer, J.; Hallmans, G.; Borena, W.; Häggström, C.; Engeland, A.; Almquist, M. Metabolic risk factors and cervical cancer in the metabolic syndrome and cancer project (Me-Can). Gynecol. Oncol. 2012, 125, 330-335. [CrossRef]

52. WHO (World Health Organization) Human Papillomavirus (HPV) and Cervical Cancer. 2018. Available online: www.who.int/ (accessed on 25 January 2019).

53. Lei, J.; Ploner, A.; Lagheden, C.; Eklund, C.; Kleppe, S.N.; Andrae, B.; Elfström, K.M.; Dillner, J.; Sparén, P.; Sundström, K. High-risk human papillomavirus status and prognosis in invasive cervical cancer: A nationwide cohort study. PLoS Med. 2018, 15, e1002666. [CrossRef]

54. Hooi, D.J.; Lissenberg-Witte, B.I.; de Koning, M.N.; Pinedo, H.M.; Kenter, G.G.; Meijer, C.J.; Quint, W.G. High prevalence of high-risk HPV genotypes other than 16 and 18 in cervical cancers of Curaçao: Implications for choice of prophylactic HPV vaccine. Sex Transm. Infect. 2018, 94, 263-267. [CrossRef] [PubMed]

55. Tao, L.; Han, L.; Li, X.; Gao, Q.; Pan, L.; Wu, L.; Luo, Y.; Wang, W.; Zheng, Z.; Guo, X. Prevalence and risk factors for cervical neoplasia: A cervical cancer screening program in Beijing. BMC Public Health 2014, 14, 1185. [CrossRef] [PubMed]

56. Hwang, J.H.; Lee, J.K.; Kim, T.J.; Kim, M.K. The association between fruit and vegetable consumption and HPV viral load in high-risk HPV-positive women with cervical intraepithelial neoplasia. Cancer Causes Control 2010, 21, 51-59. [CrossRef]

57. Carstensen, B.; Plummer, M.; Laara, E.; Hills, M. Epi: A Package for Statistical Analysis in Epidemiology, R Package Version 2.35; 2019. Available online: https:/ /CRAN.R-project.org/package=Epi (accessed on 2 March 2019).

58. Team, R.C. R: A Language and Environment for Statistical Computing; R Foundation for Statistical Computing: Vienna, Australia, 2015.

59. Pohlert, T. The Pairwise Multiple Comparison of Mean Ranks Package (PMCMR). R Package. 2014. Available online: http:/ /CRAN.R-project.org/package=PMCMR (accessed on 3 February 2019).

60. Chong, J.; Soufan, O.; Li, C.; Caraus, I.; Li, S.; Bourque, G.; Wishart, D.S.; Xia, J. MetaboAnalyst 4.0: Towards more transparent and integrative metabolomics analysis. Nucleic Acids Res. 2018, 2, W486-W494. [CrossRef] [PubMed]

61. Wickham, H. ggplot2: Elegant Graphics for Data Analysis; Springer: New York, NY, USA, 2016. 